\title{
Vector Operators for Color Image Zooming
}

\author{
Rastislav Lukac*, Konstantinos N. Plataniotis*, Bogdan Smolka ${ }^{\dagger}$, Anastasios N. Venetsanopoulos* \\ ${ }^{*}$ University of Toronto, The Edward S. Rogers Sr. Department of ECE \\ 10 King's College Road, Toronto, M5S 3G4 Ontario, Canada \\ ${ }^{\dagger}$ Department of Automatic Control, Silesian University of Technology, \\ Akademicka 16 Str., 44-101 Gliwice, Poland
}

\begin{abstract}
This paper presents a vector filtering framework for color image zooming. Popular vector filters such as the vector median filter, the basic vector directional filter and the data-adaptive vector filter are used to enlarge the spatial resolution of the full-color, Red-Green-Blue images. Extensive experimentation reported int this paper indicates that the proposed color image zoomers preserve the spectral and spatial characteristics of the processed image and produce enlarged images without color shifts and artifacts.
\end{abstract}

\section{INTRODUCTION}

The commercial proliferation of imaging sensor based color image processing systems and their use in novel applications such as wireless phones, pocket devices, surveillance, and automotive apparatus have increased the demand for new algorithmic and technical developments in the area of color imaging [1]. Modern color image processing systems use processing operators which rely on the trichromatic theory of color (Fig.1) [2]. Since the cost of imaging system rapidly increases with optical zooming capturing capabilities, to keep it at a reasonable level, camera manufacturers produce cameras capable of performing digital zooming [3]. An image zooming technique performs spatial interpolation which involves increasing the apparent resolution of an image by increasing the number of pixels representing the visual data [1]. Such a zooming process (Fig.2) [3]: i) enlarges the spatial resolution of the input image, ii) completes the missing data from the spatially adjacent data using some type of the interpolation operations, and iii) preserves the spectral representation of the input image.

Spatial interpolation of a color image is required for tasks such as zooming and displaying on high resolution display devices, and increasing the spatial resolution of a digital camera output [1],[3]. Since a typical natural image exhibits significant spectral correlation among its RGB color planes, scalar techniques performed on the individual color channels are insufficient since the correlation between the channels is not considered producing thus various spectral artifacts and color shifts [1],[3]. Moreover, many conventional methods such as bilinear interpolation and spline based techniques listed in [4][6] often cause excessive blurring or geometric artifacts [3],[7]. Therefore, the development of the more sophisticated vector based, nonlinear approaches is of paramount importance.

\section{COLOR IMAGING BASICS}

Let us consider, a $K_{1} \times K_{2}$ Red-Green-Blue (RGB) color image $\mathrm{x}: Z^{2} \rightarrow Z^{3}$ representing a two-

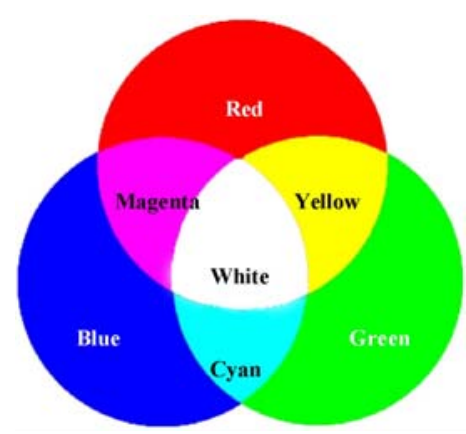

Fig. 1. Additive color mixing using Red, Green and Blue primary colors.

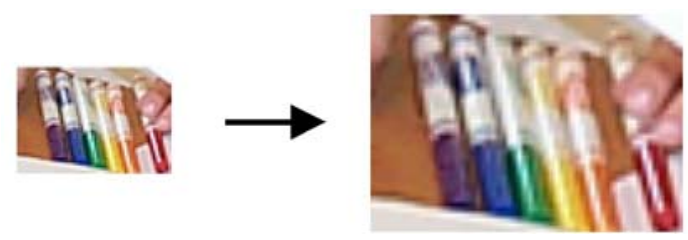

Fig. 2. Color image zooming.

dimensional matrix of three-component samples $\mathbf{x}_{(p, q)}=$ $\left[x_{(p, q) 1}, x_{(p, q) 2}, x_{(p, q) 3}\right]$. In a given RGB image, pixel $\mathbf{x}_{(p, q)}$ denotes the color vector occupying the spatial location $(p, q)$ with the coordinates $p=1,2, \ldots, K_{1}$ and $q=1,2, \ldots, K_{2}$. The component $x_{(p, q) k}$, for $k=1,2,3$, represent the $k$-th elements of $\mathbf{x}_{(p, q)}$ with $k=1$ indicating the $\mathrm{R}$ component, $k=2$ indicating the $\mathrm{G}$ component, and $k=3$ denoting the $\mathrm{B}$ component.

Each color pixel $\mathbf{x}_{(p, q)}$ is a three-dimensional vector, uniquely defined by its length (magnitude) $M_{\mathrm{x}}: Z^{2} \rightarrow$ $R^{+}$and orientation (direction) $D_{\mathrm{x}}: Z^{2} \rightarrow S^{2}$ in the vector space, [1]. The magnitude

$$
M_{\mathbf{x}_{(p, q)}}=\left\|\mathbf{x}_{(p, q)}\right\|=\sqrt{x_{(p, q) 1}^{2}+x_{(p, q) 2}^{2}+x_{(p, q) 3}^{2}}
$$

of the color vector relates to the luminance, whereas the vectors' directionality

$$
D_{\mathbf{x}_{(p, q)}}=\frac{1}{\left\|\mathbf{x}_{(p, q)}\right\|} \mathbf{x}_{(p, q)}=\frac{1}{M_{\mathbf{x}_{(p, q)}}} \mathbf{x}_{(p, q)}
$$

with $S^{2}$ denoting a unit ball in $R^{3}$ and $\left\|D_{\mathbf{x}_{(p, q)}}\right\|=1$, relates to the chromaticity characteristics of the pixel.

Since both measures are essential for human perception, any color image processing solution should process the color pixels as a set of vectors to preserve both magnitude and directional characteristics of the color vectors [1],[8]. 


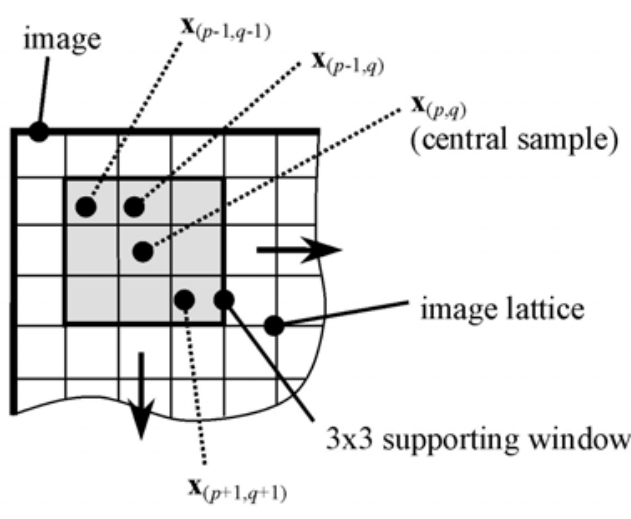

Fig. 3. Arrangements of the vectorial inputs in the supporting window $W$ sliding over the image domain.

\section{Vector ZoOming of COLOR IMAGES}

The most popular vector image processing techniques, such as those listed in [1],[8], operate on some type of sliding window $W_{(p, q)}=\left\{\mathbf{x}_{(i, j)} ;(i, j) \in \zeta\right\}$ (Fig.3) which usually affects the sample $\mathbf{x}_{(p, q)}$ placed in the center of the window, changing its value by some function $f(\cdot)$ of a local neighborhood area $W_{(p, q)}$ with by the area of support $\zeta$. This window operator slides over the image to calculate individually all the new image pixels in the enlarged color image.

Zooming the color data $\mathbf{x}_{(p, q)}$ by a factor of $z$ results in a $z K_{1} \times z K_{2}$ zoomed color image color image $\mathbf{x}^{\prime}$ : $Z^{2} \rightarrow Z^{3}$ The zooming factor $z \in Z$ can be an arbitrary positive integer, however, the value $z=2$ is selected here to facilitate the discussion. Assuming the aforementioned setting, the use of the zooming procedure maps the original color vectors $\mathbf{x}_{(p, q)}$ with spatial coordinates $p$ and $q$ into the enlarged image $\mathbf{x}^{\prime}$ as follows [3]:

$$
\mathbf{x}_{(2 p-1,2 q-1)}^{\prime}=\mathbf{x}_{(p, q)}
$$

where the pixels $\mathbf{x}_{(2 p, 2 q)}^{\prime}$ denote the new rows and columns (e.g. of zeros) added to the original data.

Using a $3 \times 3$ processing window (Fig.3) on the upsampled image, the three pixel configurations (Figs.4a$c$ ) are obtained when the window is centered on an empty pixel position. Configurations depicted in Figs. 4 b,c provide an insufficient number of original pixels for the estimation of the unknown vector at the center of the window. Therefore, a two-iteration interpolation procedure is employed. Assuming that $(p, q)$, for $p=1,2, \ldots, 2 K_{1}$ and $q=1,2, \ldots, 2 K_{2}$, denotes the spatial position of the estimated color vector $\mathbf{x}_{(p, q)}^{\prime}$ in the enlarged color image $\mathbf{x}^{\prime}$, the first interpolation step requires positioning the window in all (even $p$, even $q$ ) locations to achieve all possible configurations similar to the one depicted in Fig.4a. The unknown vector $\mathrm{x}_{(p, q)}^{\prime}$ is estimated using some operator operating over the four known pixels in $W=\left\{\mathbf{x}_{(i, j)}^{\prime}\right.$, for $\left.(i, j) \in \zeta\right\}$ with $\zeta=\{(p-1, q-$ $1),(p-1, q+1),(p+1, q-1),(p+1, q+1)\}$. After this interpolation step, all configurations become similar to the one depicted in Fig. $4 d$ with $\zeta=\{(p-1, q),(p, q-$ $1),(p, q+1),(p+1, q)\}$. Thus, the second interpolation

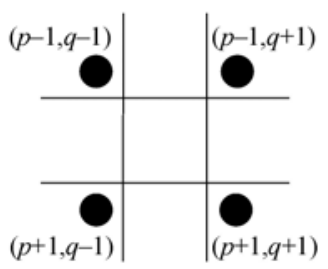

(a)

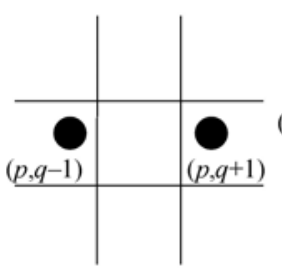

(c)

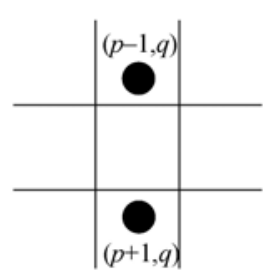

(b)

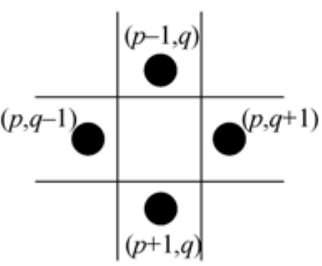

(d)
Fig. 4. Spatial arrangements of available color vectors during the zooming procedure.

step is performed on all remaining positions (odd $p$, even $q$ ) and (even $p$, odd $q$ ) with unknown pixels $\mathbf{x}_{(p, q)}^{\prime}$ located in the center of the processing window $W$ by using an operator on the two known and two previously estimated pixels within the window. This processing step completes the spatial interpolation process resulting in the fullypopulated, enlarged color image $\mathbf{x}^{\prime}$.

It is not difficult to see that by replacing the interpolation function $f\left(W_{(p, q)}\right)$, the vector processing framework can offer solutions which differ in the design philosophy, characteristics, computational complexity and performance. To demonstrate the suitability of the vector processing solutions the vector median filter (VMF) [9], the vector directional filter (VDF) [10], and the vector data-adaptive filters (VDAF) [11] are described and used in the sequence for comparison purposes.

\section{A. A Vector Median Filter}

The output $\mathbf{x}_{(p, q)}^{\prime}$ of the VMF scheme [9] is expressed as the sample $\mathbf{x}_{(i, j)}^{\prime} \in W_{(p, q)}$ minimizing the distance to other samples inside the processing window $W_{(p, q)}$ :

$$
\mathbf{x}_{(p, q)}^{\prime}=\min \underset{\mathbf{x}_{(i, j)}^{\prime} \arg }{ } \sum_{(g, h) \in \zeta}\left\|\mathbf{x}_{(i, j)}^{\prime}-\mathbf{x}_{(g, h)}^{\prime}\right\|_{L}
$$

where $\left\|\mathbf{x}_{(g, h)}^{\prime}-\mathbf{x}_{(i, j)}^{\prime}\right\|_{L}$ is the Minkowski metric [12] determining the distance between two color vectors $\mathbf{x}_{(g, h)}^{\prime}$ and $\mathbf{x}_{(i, j)}^{\prime}$ :

$$
\left\|\mathbf{x}_{(i, j)}^{\prime}-\mathbf{x}_{(g, h)}^{\prime}\right\|_{L}=\left(\sum_{k=1}^{3}\left|x_{(i, j) k}^{\prime}-x_{(g, h) k}^{\prime}\right|^{L}\right)^{\frac{1}{L}}
$$

where $L$ denotes the norm parameter, e.g. the city-block distance $(L=1)$ or Euclidean distance $(L=2)$.

The output of the VMF filter can be equivalently determined using the vector order-statistics. By calculating the aggregated distance $D_{(i, j)}$ :

$$
D_{(i, j)}=\sum_{(g, h) \in \zeta}\left\|\mathbf{x}_{(i, j)}^{\prime}-\mathbf{x}_{(g, h)}^{\prime}\right\|_{2}
$$


the ordered set of the aggregated distances can be written as follows:

$$
D_{(1)} \leq D_{(2)} \leq \ldots \leq D_{(N)}
$$

Assuming that the ordering of $D_{(i, j)}$ 's implies the same ordering of the corresponding vectors $\mathbf{x}_{(i, j)}^{\prime}$, for $(i, j) \in \zeta$, the procedure reports an ordered set of vectors

$$
\mathbf{x}_{(1)} \leq \mathbf{x}_{(2)} \leq \ldots \leq \mathbf{x}_{(N)}
$$

where $\mathbf{x}_{(r)} \in W_{(p, q)}$, for $r=1,2, \ldots, N$, denotes the $r$-th vector order-statistics [1],[2],[8]. The lowest ranked vector $\mathbf{x}_{(1)}$ is associated with the minimum aggregated distances $D_{(1)} \in\left\{D_{(i, j)}\right.$, for $\left.(i, j) \in \zeta\right\}$ and the uppermost ranked vector $\mathbf{x}_{(N)}$ corresponds to the maximum aggregated distances $D_{(N)} \in\left\{D_{(i, j)}\right.$, for $\left.(i, j) \in \zeta\right\}$. Since (6) expresses the similarity of vector $\mathbf{x}_{(i, j)}^{\prime}$ to other vectors inside $W_{(p, q)}$, the lowest ranked vector $\mathbf{x}_{(1)} \in W_{(p, q)}$ is the most typical sample for the vectorial set $W_{(p, q)}$ and therefore is considered here as the zoomer output. Thus, the VMF interpolation function $f(\cdot)$ produces the output which is restricted to the dynamic range of the input samples and thus, it never introduces new samples. The selective nature of the VMF operator and the use of the minimization concept ensure the outputting of the color vector which is the most similar, under the aggregated distance criterion (6), to other color vectors in $W_{(p, q)}$.

\section{B. A Basic Vector Directional Filter}

Another class of vector operators, termed VDF filters [10], is operating on the directional domain of color images. The BVDF output $\mathbf{x}_{(p, q)}^{\prime}$ is the sample $\mathbf{x}_{(i, j)}^{\prime} \in$ $W_{(p, q)}$ which minimizes the angular ordering criteria to other samples inside the sliding filtering window $W_{(p, q)}$ :

$$
\mathbf{x}_{(p, q)}^{\prime}=\min \underset{\mathbf{x}_{(i, j)}^{\prime} \arg }{\sum_{(g, h) \in \zeta}} \mathrm{A}\left(\mathbf{x}_{(i, j)}^{\prime}, \mathbf{x}_{(g, h)}^{\prime}\right)
$$

where

$$
\mathrm{A}\left(\mathbf{x}_{(i, j)}^{\prime}, \mathbf{x}_{(g, h)}^{\prime}\right)=\cos ^{-1}\left(\frac{\mathbf{x}_{(i, j)}^{\prime} \cdot \mathbf{x}_{(g, h)}^{\prime}}{\left|\mathbf{x}_{(i, j)}^{\prime}\right|\left|\mathbf{x}_{(g, h)}^{\prime}\right|}\right)
$$

represents the angle between two color vectors $\mathbf{x}_{(i, j)}^{\prime}$ and $\mathbf{x}_{(g, h)}^{\prime}$.

Similarly as in the VMF scheme, the BVDF filter can be equivalently expressed through the order-statistic approach [1]. Assuming that each vectorial input is associated with the aggregated angular measure

$$
\alpha_{(i, j)}=\sum_{(g, h) \in \zeta} \mathrm{A}\left(\mathbf{x}_{(i, j)}^{\prime}-\mathbf{x}_{(g, h)}^{\prime}\right)
$$

and the ordering of $\alpha_{(i, j)}$ 's implies the same ordering of the input vectors $\mathbf{x}_{(i, j)}^{\prime}$, for $(i, j) \in \zeta$, the sample $\mathbf{x}_{(1)}$ in (8), i.e. the lowest ranked vector or the lowest order-statistics, associated with the minimum aggregated angular measure $\alpha_{(1)}$ is the BVDF output.

The BVDF interpolation function $f(\cdot)$ ensures the outputting of the color vector which is the most similar, under the aggregated angular criterion (11), to other color vectors in $W_{(p, q)}$. The angular minimization approach is useful for directional data such as color data [1]. Therefore, the filtering schemes based on directional processing of color images (or directional processing followed by magnitude processing) [1],[2],[10] may achieve better performance than the VMF based approaches in terms of the color chromaticity (direction of color data) preservation.

\section{A Data-Adaptive Filter}

To proportionally represent the contribution of the adjacent color vectors $\mathbf{x}_{(i, j)}^{\prime}$, for $(i, j) \in \zeta$, in the calculation of $\mathbf{x}_{(p, q)}^{\prime}$, an averaging operation may be required to produce a suitable vector representative for the local image area $W_{(p, q)}$. Adopting a VDAF framework in [11], the interpolated color vector $\mathbf{x}_{(i, j)}^{\prime}$ is obtained as follows:

$$
\mathbf{x}_{(p, q)}^{\prime}=f\left(\sum_{(i, j) \in \zeta} w_{(i, j)}^{\prime} \mathbf{x}_{(i, j)}^{\prime}\right)
$$

where $f(\cdot)$ is a nonlinear function that operates over the weighted average of the input set. The normalized weights $w_{(i, j)}^{\prime}=w_{(i, j)} / \sum_{(i, j) \in \zeta} w_{(i, j)}$ is obtained using the filter weights $w_{(i, j)}$, where each $w_{(i, j)}$ is associated with the input color vector $\mathbf{x}_{(i, j)}^{\prime}$. The normalized weights $w_{(i, j)}^{\prime}$ provide the degree to which an input vector contributes to the output of the filter. Since the solution is unbiased, (12) must satisfy two conditions [11]: i) $w_{(i, j)}^{\prime} \geq 0$, and ii) $\sum_{(i, j) \in \zeta} w_{(i, j)}^{\prime}=1$.

Operating on the vectorial inputs $\mathbf{x}_{(i, j)}^{\prime}$, the weights $w_{(i, j)}$ of (12) are determined adaptively using functions of a distance criterion between the input vectors. Since the relationship between distances measured in physical units and perception is generally exponential [11], an exponential type of function maybe suitable to be used in the weighting formulation. Utilizing the sigmoidal membership function and the aggregated chrominance (angular) criteria $\alpha_{(i, j)}$ of (11), the weight adaptation in (12) is performed as follows:

$$
w_{(i, j)}=\beta\left(1+\exp \left\{\alpha_{(i, j)}\right\}\right)^{-r}
$$

where $\beta$ is a normalizing constant and $r$ is a parameter adjusting the weighting effect of the membership function. Based on the aggregated Minkowski metrics $D_{(i, j)}$ of (6), the adaptation formula (13) is redefined as follows:

$$
w_{(i, j)}=\beta\left(1+\exp \left\{D_{(i, j)}\right\}\right)^{-r}
$$

Thus, the VDAF can operate either on magnitude or direction of the vectorial inputs. Since $\alpha_{(i, j)}$ and $D_{(i, j)}$ significantly differs in the values, (13) and (14), respectively, produce different weights and the filtering scheme (12) will result in two different outputs.

\section{EXPERIMENTAL RESUlts}

The proposed vector zoomers are tested using the color images shown in Fig.5. The database contains natural, real life images which vary in complexity and color appearance. Note that in order to facilitate comparisons, all images have been normalized to the standard $512 \times 512$, 8-bit per channel RGB representation. 


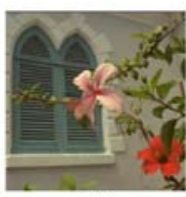

(a)

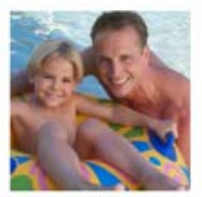

(b)

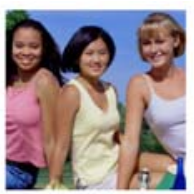

(c)

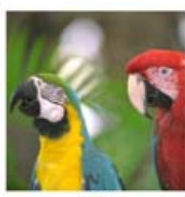

(d)
Fig. 5. Test color images: (a) Window, (b) Water, (c) Girls, (d) Parrots.

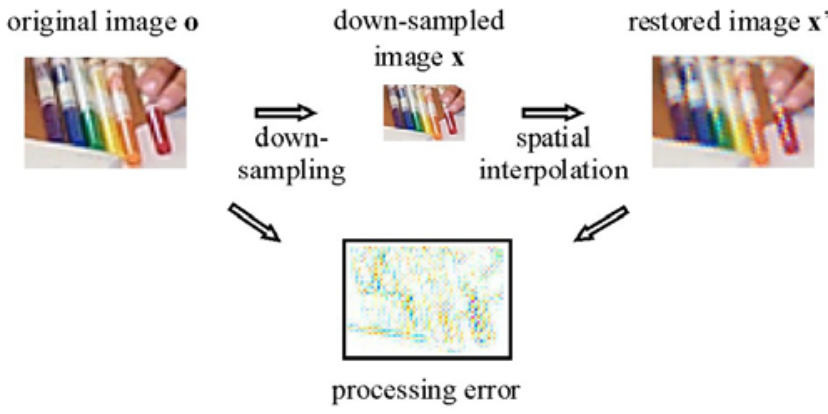

Fig. 6. Evaluation procedure.

Fig.6 visualizes the evaluation procedure used in this experimentation. To measure the efficiency of the zooming methods objectively, the process starts with the $z K_{1} \times$ $z K_{2}$ original color image $\mathbf{o}$ which is down-sampled with a factor of $z=2$ to produce the $K_{1} \times K_{2}$ color image $\mathbf{x}$. Upon completion of the down-sampling step, the image $\mathrm{x}$ is then transformed via (3) into the $z K_{1} \times z K_{2}$ image $\mathbf{x}^{\prime}$. Using the color image zooming method we can obtain the zoomed $z K_{1} \times z K_{2}$ Bayer image $\mathbf{x}^{\prime}$. Comparative evaluations are performed by comparing the original color images $\mathbf{o}$ to interpolated images $\mathbf{x}^{\prime}$. The difference between $\mathbf{o}$ and $\mathbf{x}^{\prime}$ constitutes the error image $\left\|\mathbf{o}-\mathbf{x}^{\prime}\right\|$, which is the result of the zoomer's inaccuracy. This difference is measured using both objective and subjective criteria.

To measure the similarity between the original $\mathbf{o}$ and interpolated image $\mathrm{x}^{\prime}$ with the spatial dimensions of $z K_{1} \times z K_{2}$ pixels, a number of different objective measures based on the difference in statistical distributions of the pixel values can be utilized. In this paper, the mean absolute error (MAE), the mean square error (MSE) and the normalized color-difference (NCD) criterion are considered [2],[3],[8].

The MAE and MSE criteria are defined as follows:

$$
\begin{aligned}
& \mathrm{MAE}=\frac{1}{3 z K_{1} z K_{2}} \sum_{k=1}^{3} \sum_{p=1}^{z K_{1}} \sum_{q=1}^{z K_{2}}\left|o_{(p, q) k}-x_{(p, q) k}^{\prime}\right| \\
& \mathrm{MSE}=\frac{1}{3 z K_{1} z K_{2}} \sum_{k=1}^{3} \sum_{p=1}^{z K_{1}} \sum_{q=1}^{z K_{2}}\left(o_{(p, q) k}-x_{(p, q) k}\right)^{2}
\end{aligned}
$$

where $\mathbf{o}_{(p, q)}=\left[o_{(p, q) 1}, o_{(p, q) 2}, o_{(p, q) 3}\right]$ is the original pixel, $\mathbf{x}_{(p, q)}^{\prime}=\left[x_{(p, q) 1}^{\prime}, x_{(p, q) 2}^{\prime}, x_{(p, q) 3}^{\prime}\right]$ is the restored pixel with $(p, q)$ denoting a spatial position in a $z K_{1} \times$ $z K_{2}$ color image and $k$ characterizing the color channel.

To quantify the perceptual similarity between the orig-
TABLE I

OBJECTIVE RESULTS CORRESPONDING TO Figs. $5 a, b$.

\begin{tabular}{|c|ccc|ccc|}
\hline Image & \multicolumn{3}{|c|}{ Window } & \multicolumn{3}{c|}{ Water } \\
\hline Method & $M A E$ & $M S E$ & $N C D$ & $M A E$ & $M S E$ & $N C D$ \\
\hline VMF & 6.106 & 128.1 & 0.0356 & 3.252 & 40.7 & 0.0281 \\
VDF & 6.185 & 133.7 & 0.0357 & 3.411 & 43.3 & 0.0281 \\
VDAF, (13) & 5.450 & 88.7 & 0.0316 & 2.927 & 26.9 & 0.0244 \\
VDAF, (14) & 5.439 & 88.3 & 0.0315 & 2.922 & 26.8 & 0.0243 \\
\hline
\end{tabular}

TABLE II

OBJECTIVE RESULTS CORRESPONDING TO Figs. $5 c, d$.

\begin{tabular}{|c|rrr|rrr|}
\hline Image & \multicolumn{3}{|c|}{ Girls } & \multicolumn{3}{c|}{ Parrots } \\
\hline Method & $M A E$ & \multicolumn{1}{|c}{$M S E$} & $N C D$ & $M A E$ & $M S E$ & \multicolumn{1}{c|}{ NCD } \\
\hline VMF & 4.863 & 110.7 & 0.0467 & 4.273 & 99.0 & 0.229 \\
VDF & 4.961 & 113.8 & 0.0468 & 4.406 & 104.3 & 0.0231 \\
VDAF, (13) & 4.585 & 78.4 & 0.0457 & 4.042 & 73.0 & 0.0210 \\
VDAF, (14) & 4.573 & 78.0 & 0.0457 & 4.030 & 72.2 & 0.0210 \\
\hline
\end{tabular}

inal and the obtained solution, the NCD criterion is used:

$$
\mathrm{NCD}=\frac{\sum_{p=1}^{z K_{1}} \sum_{q=1}^{z K_{2}} \sqrt{\sum_{k=1}^{3}\left(\bar{o}_{(p, q) k}-\bar{x}_{(p, q) k}^{\prime}\right)^{2}}}{\sum_{p=1}^{z K_{1}} \sum_{q=1}^{z K_{2}} \sqrt{\sum_{k=1}^{3}\left(\bar{o}_{(p, q) k}\right)^{2}}}
$$

where $\overline{\mathbf{o}}_{(p, q)}=\left[\bar{o}_{(p, q) 1}, \bar{o}_{(p, q) 2}, \bar{o}_{(p, q) 3}\right]$ and $\overline{\mathbf{x}}_{(p, q)}^{\prime}=$ $\left[\bar{x}_{(p, q) 1}^{\prime}, \bar{x}_{(p, q) 2}^{\prime}, \bar{x}_{(p, q) 3}^{\prime}\right]$ are the vectors representing the RGB vectors $\mathbf{o}$ and $\mathbf{x}^{\prime}$, respectively, in the CIE LUV color space [2].

Table I and Table II summarize the objective results produced by the presented vector filters. Due to the adaptive nature the VDAF solutions outperform the VMF and VDF solutions. The improvement achieved by using the VDAF should also be addressed to the weighted average type of the operator which allows to proportionally represent the contribution of the neighboring, available color vectors in the zoomer's input. Visual inspection of the results presented in Fig.7 reveals that the VMF and BVDF are able to maintain sharp edges, however, these schemes produce the so-called streaks (regions of similar intensity) which deteriorate the visual quality. The VDAF outputs are not as sharp but provides an excellent tradeoff between sharpness and the reduction of the streaks' effect. Finally, Fig.8 allows the visual comparison of the images restored by the VMF and the scalar (componentwise) MF [13]. These results illustrate that the scalar approach produces color shifts and artifacts whereas the vector approach preserves both the spatial and spectral characteristics of the enlarged image. Note that although each spatial interpolation introduces processing errors to the enlarged image, a significant portion of introduced spatial artifacts (not color artifacts which are caused by the lack of spectral information in the MF scheme) in the images shown in Figs.7-8 should be attributed to the down-sampling process used in the objective evaluation scenario (Fig.6) [3]. Therefore, visually more pleasing images can be obtained by applying image zoomers to the 


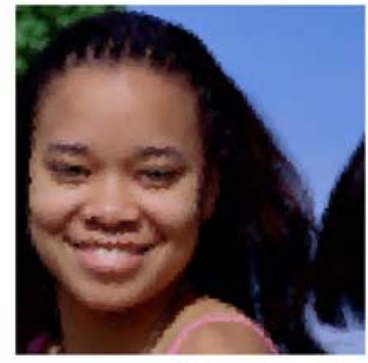

(a)

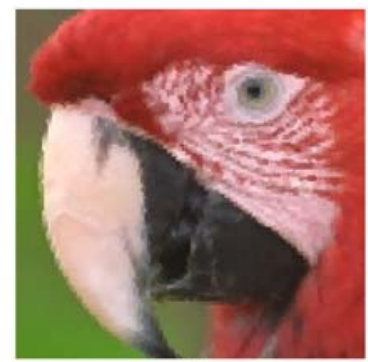

(e)

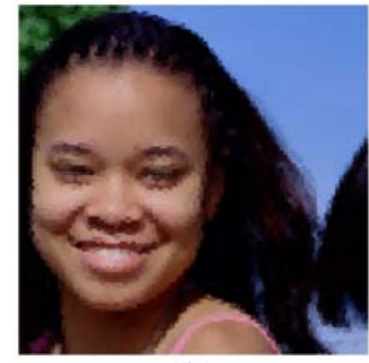

(b)

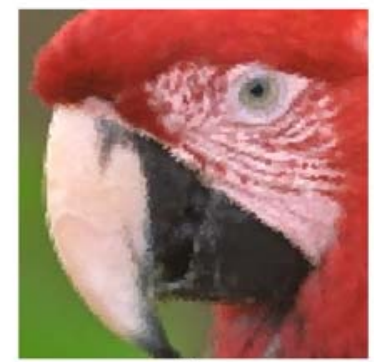

(f)

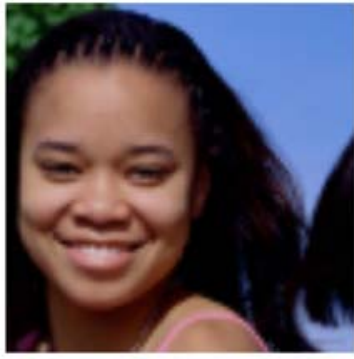

(c)

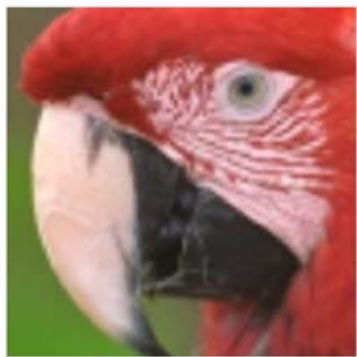

(g)

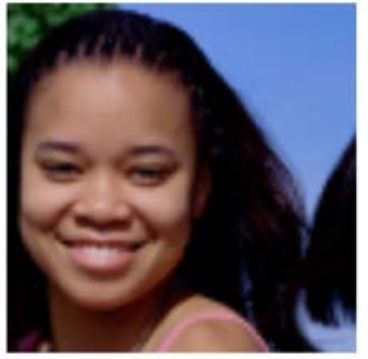

(d)

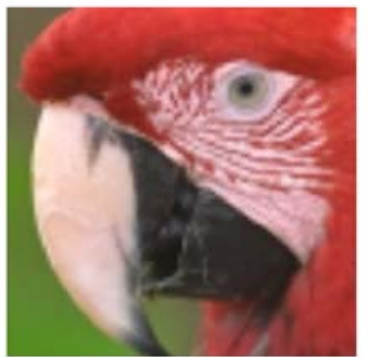

(h)

Fig. 7. Obtained results: (a,e) VMF, (b,f) VDF, (c,g) VDAF with (13), (d,h) VDAF with (14).

original images o. Finally, it should be noted that the cost of vector filters has been find reasonable in various applications. The interesting reader should refer to [2],[8],[14] for computational complexity analysis. Results indicate that the adaptive vector zoomers may be of interest when it comes to enlarge the color images.

\section{CONCLUSION}

A vector processing framework for color image zooming was presented. The vector methods utilize the inherent spectral correlations that exists between color planes of the natural RGB images and process the color pixels as the set of color vectors. Therefore, the vector processing based methods preserve both spatial and spectral characteristics of the processed color image.

\section{REFERENCES}

[1] R. Lukac, B. Smolka, K. Martin, K. N. Plataniotis, and A. N. Venetsanopulos, "Vector filtering for color imaging," IEEE Signal Processing Magazine; Special Issue on Color Image Processing, vol. 22, January 2005.

[2] K. N. Plataniotis and A. N. Venetsanopoulos, Color Image Processing and Applications, Springer Verlag, 2000.

[3] R. Lukac, K. N. Plataniotis, and D. Hatzinakos, "Color image zooming on the Bayer pattern," IEEE Transactions on Circuit and Systems for Video Technology, to appear, vol. 15, 2005.

[4] R. G. Keys, "Cubic convolution interpolation for digital image processing," IEEE Transactions on Acoustics, Speech and Signal Processing, vol. 29, pp. 1153-1160, December 1981.

[5] S. E. Reichenbach, and F. Geng, "Two-dimensional cubic convolution," IEEE Transactions on Image Processing, vol. 12, pp.857-865, August 2003.

[6] S. Battiato, G. Gallo, and F. Stanco, "A locally adaptive zooming algorithm for digital images," Image and Vision Computing, vol. 20, pp. 805-812, September 2002.

[7] N. Herodotou and A. N. Venetsanopoulos, "Colour image interpolation for high resolution acquisitions and display devices," IEEE Transaction on Consumer Electronics, vol. 41, pp. 1118-1126, November 1995.

[8] R. Lukac, K. N. Plataniotis, B. Smolka, and A. N. Venetsanopoulos: "Generalized selection weighted vector filters," EURASIP Journal on Applied Signal Processing: Special Issue on Nonlinear Signal and Image Processing, vol. 2004, pp. 1870-1885, October 2004. (a)
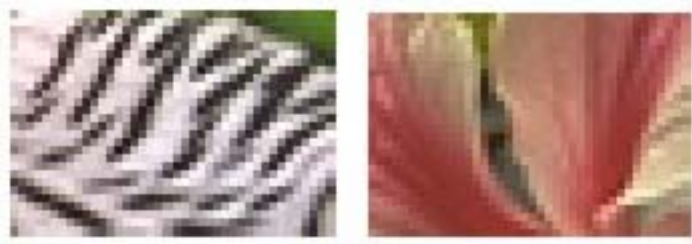

(b)

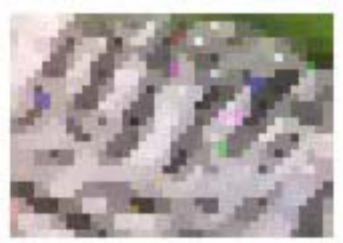

(c)
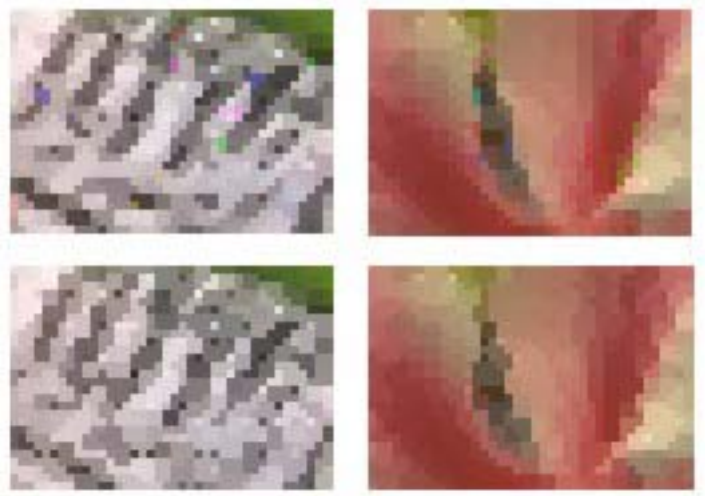

Fig. 8. Scalar vs. vector MF: (a) original image, (b) MF, (c) VMF.

[9] J. Astola, P. Haavisto, and Y. Neuvo, "Vector median filters," Proceedings of the IEEE, vol. 78, pp. 678-689, April 1990.

[10] P. E. Trahanias, D. Karakos, and A. N. Venetsanopoulos, "Directional processing of color images: theory and experimental results," IEEE Trans. Image Processing, vol. 5, pp. 868-881, June 1996.

[11] K. N. Plataniotis, D. Androutsos, and A. N. Venetsanopoulos, "Adaptive fuzzy systems for multichannel signal processing," Proceedings of the IEEE, vol. 87, pp. 1601-1622, September 1999.

[12] R. O. Duda, P. E. Hart and D. G. Stork, Pattern Classification and Scene Analysis. John Wiley, Second Edition, 2000.

[13] I. Pitas and A. N. Venetsanopoulos, "Order statistics in digital image processing," Proceedings of the IEEE, vol. 80, pp. 18921919, Dec. 1992.

[14] B. Smolka, K.N. Plataniotis, and A.N. Venetsanopoulos, "Nonlinear techniques for color image processing," in Nonlinear Signal and Image Processing: Theory, Methods, and Applications, (eds.) K.E. Barner and G.R. Arce, CRC Press, pp. 445-505, 2004. 
